\title{
On the use of Shunt Impedances versus Bounded Environment Passivity for teleoperation systems
}

\author{
B. Willaert, M. Franken, H. Van Brussel and E.B. Vander Poorten
}

\begin{abstract}
This paper analyses and compares two passivitybased approaches that allow to include a-priori knowledge on the dynamic range of the human operator and/or the environment. This can lead to less conservative teleoperation systems compared to systems designed to be purely passive or absolutely stable. The first approach under investigation is a method where the absolute stability is analysed of a teleoperation system augmented with shunt impedances in series and/or parallel with the teleoperation system. It is shown that the traditional interpretation of the use of shunt impedances is not valid and a more accurate description of how to use this method is presented. The second approach under investigation is the bounded environment (operator) method. It is shown that the original idea to restrict the analysis to the so-called worst-case scenarios of a pure mass and a pure stiffness as environment can be too simplistic. Illustrative examples with mass-spring-damper systems fixed to the ground and floating objects as environments are made to demonstrate this in detail. In conclusion, this paper shows that embedding environment knowledge into the controller analysis/design is not straightforward and further research should be dedicated to determine which bounds should be used to obtain practically stable systems for different applications.
\end{abstract}

\section{INTRODUCTION}

When designing a teleoperation system, one has to find a good balance between robustness and non-conservativeness. It depends on the envisioned application how much information on the environment and/or human operator can be included in the stability analysis. The more restrictions or bounds on the environment and/or human operator are taken into account, the less conservative the resulting teleoperation system can be made, but also the less robust this teleoperation system will be. A simple example is the difference between the two-port passivity method [1], [2] and the absolute stability method [3], [4]. The absolute stability method can result in less conservative conditions as it takes into account that there is no direct interaction between the human operator and the environment. Depending on how likely the occurence of such a direct interaction is for the envisioned application, the designer has to decide if absolute stability is sufficiently robust.

In literature, there is a consensus that both of the above methods can still be too conservative. The problem is that both methods assume an infinite dynamic range for the human operator as well as for the environment, while in

B. Willaert, H. Van Brussel and E.B. Vander Poorten are with the Department of Mechanical Engineering, K.U.Leuven, Celestijnenlaan 300B, B-3001 Heverlee, Belgium

M. Franken is with the Control Engineering Laboratory, University of Twente, P.O. Box 217 , 7500 AE Enschede, The Netherlands

Corresponding author: bert.willaert@mech.kuleuven.be reality, the dynamic range of both the human operator and the environment is always bounded in some way. This paper discusses in detail the possibilities and also shortcomings of two frequency-domain passivity-based approaches that allow to include restrictions on these dynamic ranges when designing a teleoperation system. The focus in this paper lies on environment bounds for the case that the environment can be represented by a mass-spring-damper model-structure:

$$
Z_{e}(s)=\frac{F_{e}}{v_{e}}=M_{e} s+B_{e}+\frac{K_{e}}{s} .
$$

This model-structure is often used in literature for both the environment and the human operator [5], [6], [7], [8].

A first approach is to look at absolute stability of the hybrid matrix $\mathbf{H}_{\text {sh }}$, representing the teleoperation system with virtual shunt impendances at one or both interaction ports. Hashtrudi-Zaad and Salcudean proposed the use of such shunt impedances as a way to obtain more relaxed absolute stability conditions [9]. This idea was adopted by a.o. Adams and Hannaford [10], Cho and Park [11] and Vander Poorten [12] and used as a way to include the knowledge of a maximum and/or minimum impedance for the human operator and/or the environment. However, little attention was paid to the interpretation of these so-called maximum and minimum impedances in real practice. The above works use e.g. a shunt impedance $Z_{e 2}$ placed in series with the teleoperation system at the interaction side with the environment. This impedance $Z_{e 2}$, shown in Fig. 1(a), has a mass-spring-damper model-structure. Because this is called the maximum environment impedance, one would assume that this implies that the teleoperation system without the shunt is at least guaranteed stable for all mass-spring-damper environments with a set of $\left(M_{e}, B_{e}, K_{e}\right)$-values that fit in the
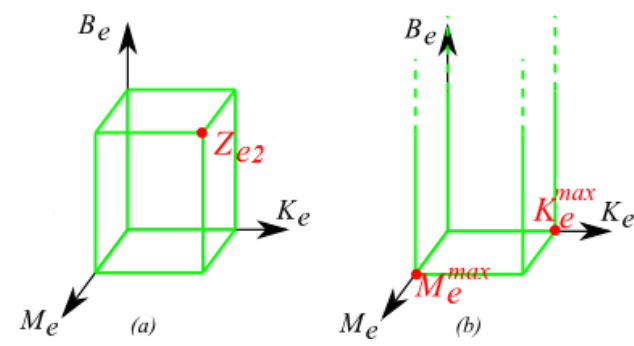

Fig. 1. Research questions: for an environment that can be represented by a mass-spring-damper model-structure: (a) does absolute stability with the shunt impedance $Z_{e 2}$ imply guaranteed stable interaction with all massspring-damper environments that fit in the box? (b) can the bounded environment passivity conditions for a pure mass and a pure stiffness be generalized to all mass-spring-damper systems which have values that fit in the box with infinite height? 
box shown in Fig. 1(a). However, a careful analysis of this approach in the first part of this paper shows that this is not the case.

A second approach looks at either the passivity of the admittance $Y_{M S}$ representing the teleoperation system including the environment, i.e. bounded environment passivity, or the passivity of the admittance $Y_{S M}$ representing the teleoperation system including the human operator, i.e. bounded operator passivity. Both these admittances are shown in Fig. 2. While Haddadi and Hashtrudi-Zaad, calculate the passivity of these one-ports numerically in the scattering domain [7], Willaert et al. proposed to calculate directly the passivity of the same one-ports and, when feasible, analytically [8]. To design a teleoperation system, it is proposed in this latter work to focus on the specific knowledge of a maximum bound for a pure stiffness as environment and the knowledge of a maximum bound for a pure mass as environment. These two bounds are shown as the red dots in Fig. 1(b). Furthermore, it is assumed that these two bounds can be extended to all mass-spring-damper systems which have values that fit in the box with infinite height. However, a counter-example for this assumption is given in the second part of the paper together with a more appropriate description of the use of the bounded environment passivity method.

To support the analysis of the above-mentioned approaches, some illustrative calculations will be done for a 1d.o.f. teleoperation system with a Position-Force controller. This system is described first in section II. In Section III, the approach of calculating absolute stability properties including shunt impedances is analyzed. Next, the bounded environment passivity method is analyzed in Section IV, after which the obtained results are discussed in Section V. Section VI concludes the paper.

\section{THE CASE STUDY}

The case study in this paper is a 1-d.o.f. teleoperation system with a specific implementation of the Position-Force controller, i.e. the PD-F scheme described in [8]. Throughout the paper, 'the Position-Force controller' refers to this specific implementation and the following equations describe the forces applied by the motors of the master and the slave:

$$
\tau_{m}=-\lambda F_{e} \text { and } \tau_{s}=\left(K_{v} s+K_{p}\right) \cdot\left(\mu x_{m}-x_{s}\right) .
$$

The parameters $\mu$ and $\lambda$ are the position and force scaling factors and $x_{m}$ and $x_{s}$ are the positions of the motors of the master and the slave, respectively. The teleoperation system analyzed here consists of a 1-d.o.f rigid body master and slave, obeying the following equations of motion:

$$
F_{h}+\tau_{m}=Z_{m} v_{m} \text { and } \tau_{s}-F_{e}=Z_{s} v_{s}
$$

with $Z_{m}$ and $Z_{s}$ representing the rigid body impedances of the master and the slave robot:

$$
Z_{m}=M_{m} s+B_{m} \text { and } Z_{s}=M_{s} s+B_{s} .
$$

The rigid body parameters and the control parameters of the real 1-d.o.f. experimental master-slave setup described in [8] will be used for the calculations reported in this paper. Table I gives an overview of these parameters.
TABLE I

PARAMETERS OF THE TELEOPERATION SYSTEM

\begin{tabular}{|cc|cc||l|ll|}
\hline Model & \multicolumn{2}{|l||}{ Controller } & \\
\hline \hline$M_{m}:$ & $0.64 \mathrm{~kg}$ & $B_{m}:$ & $3.4 \mathrm{Ns} / \mathrm{m}$ & $K_{p}: 4000 \mathrm{~N} / \mathrm{m}$ & $\mu:$ & 1 \\
$M_{s}:$ & $0.61 \mathrm{~kg}$ & $B_{s}:$ & $11 \mathrm{Ns} / \mathrm{m}$ & $K_{v}: 80 \mathrm{Ns} / \mathrm{m}(\zeta=0.81)$ & $\lambda:$ & 1 \\
\hline
\end{tabular}

\section{ABSOLUTE STABILITY AND SHUNT IMPEDANCE}

In Fig. 2, a general teleoperation system is represented as a two-port system interacting with the human operator at one side and with the environment at the other side. This twoport system can be described by a hybrid matrix $\mathbf{H}$ which gives the relationship between the efforts and flows at both interaction sides as follows:

$$
\left[\begin{array}{c}
F_{h} \\
-v_{e}
\end{array}\right]=\left[\begin{array}{ll}
h_{11} & h_{12} \\
h_{21} & h_{22}
\end{array}\right] \cdot\left[\begin{array}{l}
v_{h} \\
F_{e}
\end{array}\right] .
$$

Such a two-port system is absolutely stable if and only if there is no set of passive one-ports for which the coupled system is unstable. Stated differently, for absolute stability, the admittance $Y_{M S}$, shown in Fig. 2, should be passive for all passive impedances $Z_{e}$, which implies that the admittance $Y_{S M}$ is passive for all passive impedances $Z_{h}$ and vice-versa. In case the teleoperation system can be considered an LTIsystem, the absolute stability can be calculated based on Llewellyn's stability criteria [3], which can be expressed in terms of the parameters of the hybrid matrix $\mathbf{H}$ :

- the parameters $h_{11}$ and $h_{22}$ have no poles in the open right-half-plane (RHP),

- any poles of $h_{11}$ and $h_{22}$ on the imaginary axis are simple and have real and positive residues, and

- the inequalities

$$
\begin{gathered}
\Re\left(h_{11}\right) \geq 0, \forall \omega \\
\eta(\omega)=-\cos \left(\angle h_{12} h_{21}\right)+2 \frac{\Re\left(h_{11}\right) \Re\left(h_{22}\right)}{\left|h_{12} h_{21}\right|} \geq 1, \forall \omega .
\end{gathered}
$$

Because absolute stability assumes an infinite dynamic range for the human operator as well as for the environment, it is often considered a too conservative condition. In [9], [10], [11], [12], virtual passive shunt impedances are used as a way to include bounds on these dynamic ranges of the human operator and/or environment. Instead of calculating absolute stability of the teleoperation system itself, absolute stability is calculated for the teleoperation system including one or more passive shunt impedances. As shown in Fig.3, four passive shunt impedances can be included in the analysis. This combined system of the teleoperation system with the

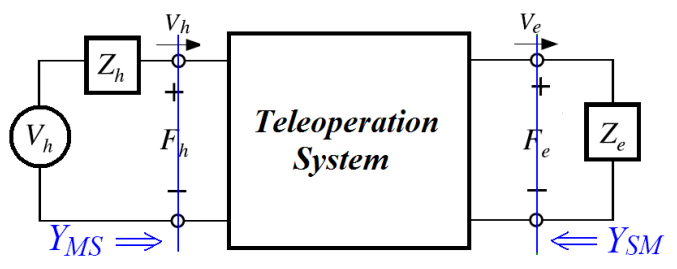

Fig. 2. A port representation of the analyzed teleoperation system. Note that $V_{h}$ represents the exogenous force input from the human operator. 
passive shunt impedances can be described by a hybrid matrix $\mathbf{H}_{\mathbf{s h}}$ which gives the relationship between the efforts and flows $F_{h}^{\prime}, v_{h}^{\prime}, F_{e}^{\prime}$ and $v_{e}^{\prime}$. If, for reasons of brevity, only the two shunt impedances at the environment side are taken into account, the hybrid matrix $\mathbf{H}_{\text {sh }}$ can be written as a function of the parameters of the hybrid matrix $\mathbf{H}$ as follows:

$$
\begin{array}{ll}
h_{11}^{s h}=h_{11}-\frac{h_{12} h_{21} Z_{e 1}}{1+h_{22} Z_{e 1}}, & h_{12}^{s h}=\frac{h_{12}}{1+h_{22} Z_{e 1}}, \\
h_{21}^{s h}=\frac{h_{21}}{1+h_{22} Z_{e 1}}, & h_{22}^{s h}=\frac{h_{22}}{1+h_{22} Z_{e 1}}+\frac{1}{Z_{e 2}}
\end{array}
$$

In [9], [10], [11], [12], $Z_{e 1}+Z_{e 2}$ is called the maximum environment impedance $Z_{e}^{\max }$ and $Z_{e 1}$ is called the minimum environment impedance $Z_{e}^{\min }$. The reasoning behind this nomenclature is the following: the impedance the teleoperation system virtually interacts with is the parallel connection of the impedance $Z_{e 1}$ and the impedance resulting from the series connection of $Z_{e 2}$ and $Z_{e}$. Thus, in the case of $Z_{e}=\infty$, the teleoperation system virtually interacts with the impedance $Z_{e 1}+Z_{e 2}$. In the case of $Z_{e}=0$, the teleoperation system virtually interacts with the impedance $Z_{e 1}$. The same holds for the operator side. This nomenclature, however, raises a first concern as the terms 'minimum' and 'maximum' impedance are in fact misleading. One can compare the stiffness or mass component of an impedance, one can compare the amplitude or the phase of an impedance at a specific frequency, but impedances as a whole cannot be compared as they are complex numbers. For the two impedances $Z_{1}=\frac{800}{s} \mathrm{Ns} / \mathrm{m}$ and $Z_{2}=3+\frac{600}{s} \mathrm{Ns} / \mathrm{m}$ e.g., one can say that the stiffness component of $Z_{1}$ is larger than that of $Z_{2}$, but saying which of both impedances is the largest is not possible. This observation forms a first concern with the approach of using passive shunt impedances: if these shunt impedances cannot be called the minimum impedance and the maximum impedance what kind of bounds are they then?

To raise a second concern with this method, the effect of the shunt impedance $Z_{e 2}$ is analyzed here in more detail. Looking at (7), one can see that, independent of $Z_{e 1}, Z_{e 2}$ only appears in the hybrid parameter $h_{22}^{s h}$. As $Z_{e 2}$ is a passive impedance, the extra poles of $h_{22}^{\text {sh }}$ always comply with the first two Llewellyn criteria. While inequality (5) is not affected by the inclusion of the shunt impedance $Z_{e 2}, \eta(\omega)$ and thus inequality (6) can be affected in case that $Z_{e 2}$ changes the positive real part of $h_{22}^{s h}$. As $\Re\left(h_{22}^{s h}\right)=\Re\left(h_{22}\right)+\Re\left(\frac{1}{Z_{e 2}}\right)$, it is clear that neither a pure mass nor a pure stiffness as shunt impedance $Z_{e 2}$ can change the positive real part of $h_{22}^{s h}$ and thus can't have any effect on the absolute stability criteria. Stated differently, the shunt impedance $Z_{e 2}$ must

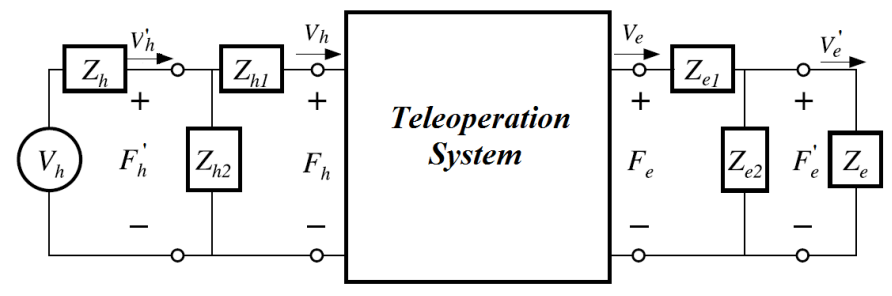

Fig. 3. A port representation of the analyzed teleoperation system when shunt impedances are present [11]. contain damping in order to affect Llewellyn's criteria. It is important to see that this finding is general and not dependent on any control structure. Note that this finding, although not explicitly, can be found in [9]. Consequently, independent of the model-structure used for the shunt impedances, damping in the shunt impedances is mandatory in order to affect the absolute stability properties of the teleoperation system including the passive shunt impedances. This observation leads to a second concern with the approach of using passive shunt impedances: if the damping is mandatory to affect the absolute stability properties of $\mathbf{H}_{\mathbf{s h}}$, how can this method be used to effectively include bounds on environments/operators that are like a pure mass or a pure stiffness?

In order to get to the right interpretation of the use of shunt impedances, again the special case that $Z_{e 1}=0, Z_{h 2}=\infty$ and $Z_{h 1}=0$ will be used. In that case, the following holds: when a teleoperation system in combination with the virtual shunt impedance $Z_{e 2}$ is calculated to be absolutely stable, this same teleoperation system without the shunt will be guaranteed stable only when interacting with all environments $Z_{e}^{*}$ that are a series connection of $Z_{e 2}$ and all possible passive impedances. All these impedances form a subspace $S_{Z_{e 2}}$ in the space of all passive impedances:

$$
S_{Z_{e 2}}=\left\{Z_{e}^{*} \mid Z_{e}^{*}=\left(Z_{e 2}^{-1}+Z_{e}^{-1}\right)^{-1} \text { with } Z_{e} \text { passive }\right\}
$$

The physical interpretation of this subspace, however, is not straightforward, as it cannot be linked to a group of environments with an arbitrary model-structure, but only to a group of environments which share a common impedance at their interaction interface. From the above raised concerns, one can deduce that the box shown in Fig. 1(a) is not fully covered by this subspace. Consequently, the approach of using shunt impedances cannot be used to include bounds expressed in terms of the mass, damper and stiffness when the environment/operator is assumed to be a mass-springdamper system.

To further illustrate this claim, a calculation is done for the teleoperation system described in Section II. Note that it is proven in [8] that this system, without shunts, is never absolutely stable. Assume now for the shunt impedance $Z_{e 2}$ a spring-damper system: $B_{e 2}+\frac{K_{e 2}}{s}$. The motivation for this is triple: (a) as mentioned above a damping term in the shunt impedance is mandatory to affect the absolute stability properties of $\mathbf{H}_{\text {sh }}$ with respect to $\mathbf{H}$, (b) for the Position-Force controller studied here, typically spring like environments are most problematic and (c) no mass term is added to keep the example as simple as possible.

Figure 4 shows for different values of $\left(B_{e 2}, K_{e 2}\right)$ the numerically calculated minimum value of $\eta(\omega)$ for the parameters of Table I and $\omega \in\left[0.01-10^{6}\right] \mathrm{rad} / \mathrm{s}$. The teleoperation system including the shunt impedance $Z_{e 2}$ is absolutely stable for every combination $\left(B_{e 2}, K_{e 2}\right)$ for which $\eta(\omega)_{\min }$ is 1 . The figure shows that only for a limited range of values for the damping $B_{e 2}$ and limited values for the stiffness $K_{e 2}$, the use of the shunt impedance $Z_{e 2}$ results in an absolutely stable system. More important, it clearly shows that if a system is absolutely stable when using a shunt impedance 


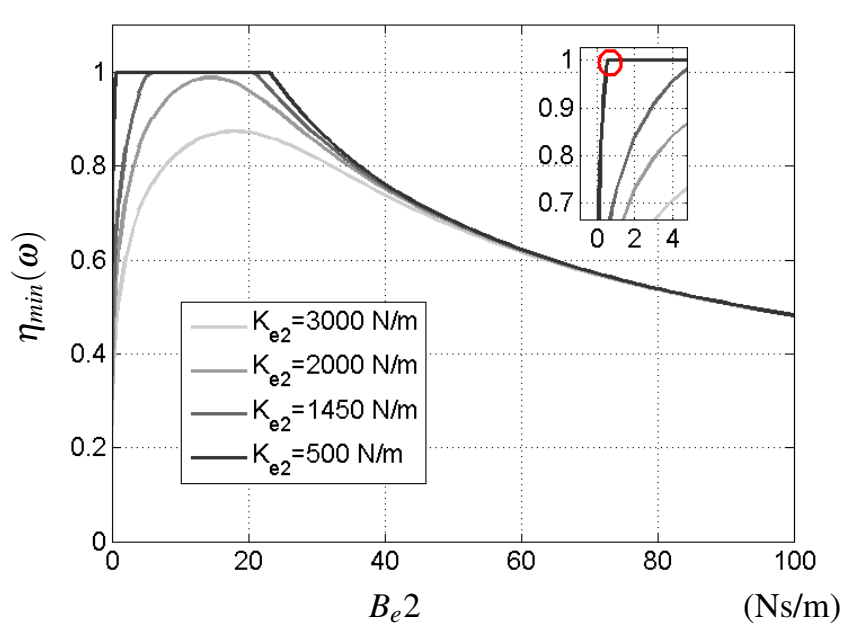

Fig. 4. The minimum value of $\eta(\omega)$ for the parameters of Table I and $\omega \in\left[0.01-10^{6}\right] \mathrm{rad} / \mathrm{s}$ for (a) a spring-damper shunt impedance at the environment side.

$Z_{e 2}=B_{e 2}+\frac{K_{e 2}}{s}$, that does not necessarily mean that the system is absolutely stable when using a shunt impedance $Z_{e 2}^{\prime}=B_{e 2}^{\prime}+\frac{K_{e 2}^{\prime}}{s}$ with $B_{e 2}^{\prime} \leq B_{e 2}$ and $K_{e 2}^{\prime} \leq K_{e 2}$. For example, when using a shunt impedance $Z_{e 2}=15+\frac{1450}{s} \mathrm{Ns} / \mathrm{m}$, the system is absolutely stable, while for $Z_{e 2}^{\prime}=2+\frac{1450}{s} \mathrm{Ns} / \mathrm{m}$ it is not, as $Z_{e 2}^{\prime}$ cannot be written as a series connection of $Z_{e 2}=15+\frac{1450}{s} \mathrm{Ns} / \mathrm{m}$ with any passive impedance, i.e. $Z_{e 2}^{\prime}$ doesn't fall in the subspace $S_{Z_{e 2}}$.

Note that, indepedent of the value for $K_{e 2}$, the value $\eta_{\min }(\omega)$ becomes smaller than one for $B_{e 2} \rightarrow 0$. This makes sense as the teleoperation system itself is not absolutely stable and damping in the shunt is mandatory to affect the absolute stability condition.

\section{BOUNDED ENVIRONMENT PASSIVITY}

The bounded environment passivity method has recently been proposed as a method that allows to incorporate bounds on the environment impedance $Z_{e}$ when designing a teleoperation system [8]. This method calculates the passivity of the admittance $Y_{M S}$, shown in Fig. 2, representing the combined dynamics of the master, the slave, the controller and the environment. Assuming an environment impedance $Z_{e}$, the admittance $Y_{M S}$ for a general teleoperation system described by the hybrid matrix $\mathbf{H}$ can be written as:

$$
Y_{M S}(s)=\frac{h_{22} Z_{e}+1}{\left(h_{22} Z_{e}+1\right) h_{11}-h_{12} h_{21} Z_{e}} .
$$

To calculate the passivity of this admittance, some assumption has to be made: which model-structure(s) can be assumed for the environment and what are the minimum and maximum values for each component of this/these modelstructure(s). Based on the idea that pure springs and pure masses are considered as worst case environments since their admittance is not strictly passive [13], Willaert et al. proposed in [8] to, depending on the application, take into account a maximum value $M_{e}^{\max }$ for a pure mass modelstructure $\left(Z_{e}=M_{e} s\right)$ and/or a maximum value $K_{e}^{\max }$ for a pure stiffness model-structure $\left(Z_{e}=\frac{K_{e}}{s}\right)$ when designing a teleoperation system. This means that the teleoperation
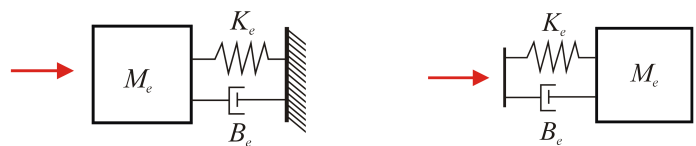

Fig. 5. Two different model-structures for the environment: (a) a massspring-damper fixed to the ground and (b) a spring-damper-mass floating object.

system has to be designed such that the following holds ${ }^{1}$ :

$$
\begin{gathered}
Y_{M S\left(M_{e}\right)} \text { is passive for } M_{e} \in\left[0, M_{e}^{\max }\right], \\
Y_{M S\left(K_{e}\right)} \text { is passive for } K_{e} \in\left[0, K_{e}^{\max }\right] .
\end{gathered}
$$

The advantage of looking at these two simple modelstructures separately, is that the number of parameters in the admittance $Y_{M S}$ is restricted which facilitates analytic calculations and the interpretation of the resulting conditions. Note that above, the bounded environment passivity method is described as a manner to use knowledge on the environment to optimize the teleoperation system (design tool). However, the method can also be used the other way around, i.e. calculating bounds on the environment for a given teleoperation system (analysis tool).

An important question, however, is whether the results obtained for this pure mass and pure stiffness as environment can be generalized to other environments. For the massspring-damper model-structure shown in Fig.5(a), the following is assumed in [8]:

ASSUMPTION 1: If $Y_{M S}$ is passive for all pure masses $M_{e} \in\left[0, M_{e}^{\max }\right]$ and all pure stiffnesses $K_{e} \in\left[0, K_{e}^{\max }\right]$, $Y_{M S}$ is guaranteed to be passive for all environments $Z_{e}=$ $M_{e} s+B_{e}+\frac{K_{e}}{s}$ with $M_{e}<M_{e}^{\max }, K_{e}<K_{e}^{\max }$ and $B_{e} \in[0, \infty]$.

This assumption corresponds to the box with infinite height shown in Fig. 1(b). However, the validity of this assumption is not proven and it will be shown below that this assumption does not generally hold. What follows now, are three different examples clarifying the use of the bounded environment passivity method by demonstrating that the obtained results cannot always be generalized.

\section{A. Position-force controller without LPF}

In this example, the bounded environment passivity method is used as a design tool. Assume that one wants to tune the controller of the teleoperation system described in Section II, for an application where the environment can be characterized by a maximum value for the pure stiffness of $600 \mathrm{~N} / \mathrm{m}$, and a maximum value for the pure mass of $0.5 \mathrm{~kg}$. The most effective tuning option ${ }^{2}$ is changing the

\footnotetext{
${ }^{1}$ As detailed in [8], the combination of BIBO-stability of $Y_{M S}$ and $Y_{M S}$ having a positive real part is a sufficient condition for passivity of $Y_{M S}$ The first requirement deals with the uncoupled stability of $Y_{M S}$, while the second requirement deals with the coupled stability of $Y_{M S}$. As typically the BIBO-stability condition is less restricting than the positive real part condition, for reasons of brevity, only the positive real part conditions are discussed here.

${ }^{2}$ As shown in [8], the position controller of the slave should be as stiff as possible. On a real setup, however, $K_{p}$ and $K_{v}$ have to be limited in order to guarantee the stability of the position loop. Thus, after the position controller is tuned as stiff as possible, changing the scaling factors is the only remaining tuning option.
} 


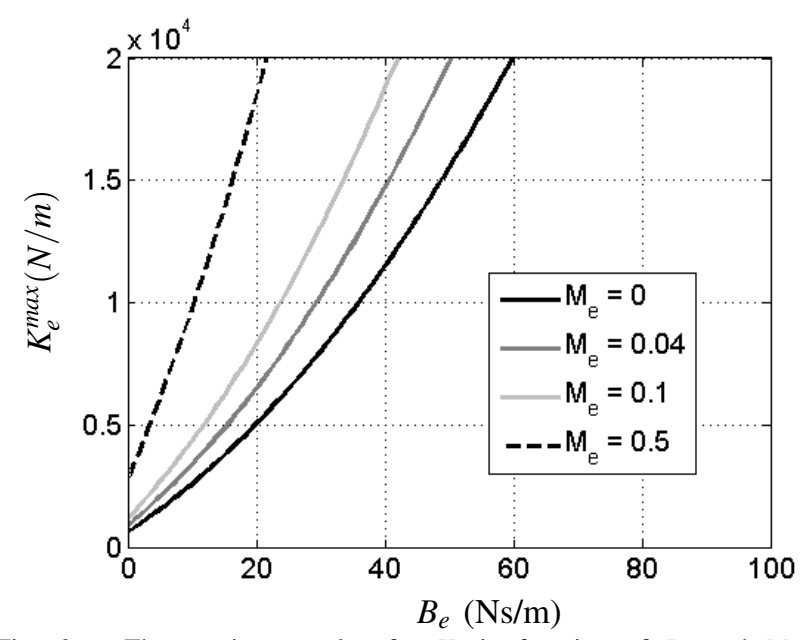

Fig. 6. The maximum value for $K_{e}$ in function of $B_{e}$ and $M_{e}$ for which $Y_{M S\left(Z_{e}\right)}$ is passive. One can see that for $M_{e}=B_{e}=0$, the maximum environment stiffness is $600 \mathrm{~N} / \mathrm{m}$.

scaling factors $\mu$ and $\lambda$. In [8] it is shown that $Y_{M S}$ is passive for all pure stiffnesses with a value smaller than $K_{e}^{\max }$ if the parameters of the teleoperation system comply with the following inequality:

$\mu \lambda \leq \frac{B_{m}\left(B_{s}+K_{v}\right)}{M_{s} K_{e}^{\max } K_{v}^{2}}\left(\left(B_{s}+K_{v}\right) K_{v}-2 M_{s} K_{p}+2 \sqrt{M_{s}^{2} K_{p}^{2}+M_{s} K_{v}^{2} K_{e}^{\max }-K_{p} K_{v} B_{s} M_{s}}\right)$.

With $K_{e}^{\max }=600 \mathrm{~N} / \mathrm{m}$, this means that $\mu \lambda$ can be maximum 0.97. Moreover, it is shown that $Y_{M S}$ is passive for all pure masses. Consequently, the condition $M_{e}^{\max }=0.5 \mathrm{~kg}$ does not result in a more restrictive tuning condition. Next, it is verified whether the above derived tuning condition $\mu \lambda \leq$ 0.97 is a sufficient condition for all mass-spring-damper systems that fit into the 'infinite' box shown in Fig. 1(b). Therefore the passivity of $Y_{M s}$ is calculated with a massspring-damper as model-structure for the environment. This is done analytically, but, since the resulting condition is very cumbersome, it is not written out here ${ }^{3}$. In Fig. 6, this condition is shown as an upper boundary on $K_{e}$, for a range of values of $B_{e}$ and some values of $M_{e}$, for the parameters of Table I and $\mu \lambda=0.97$. This figure shows that both the environment damping and the environment mass have a relaxing effect on the upper boundary for the environment stiffness. Consequently, for this teleoperation system Assumption 1 seems to hold.

\section{B. Position-force controller with LPF}

Assume now the same design task but with a $1^{s t}$-order low-pass-filter on the reflected force signal:

$$
\tau_{m}=-\lambda \frac{F_{e}}{\tau s+1} \text { and } \tau_{s}=\left(K_{v} s+K_{p}\right) \cdot\left(\mu x_{m}-x_{s}\right) .
$$

The bandwidth of the filter is $30 \mathrm{~Hz}$, i.e $\tau=0.0053$. As shown in [8], this has a negative effect on the stability properties of the system and moreover, an analytic analysis of the passivity of $Y_{M S}$ becomes too complex, as the positive real part condition, i.e. $Y_{M S} \geq 0$ for all $\omega$, results in a $4^{\text {th }}$ order

\footnotetext{
${ }^{3}$ Compared to condition for a pure stiffness as environment, i.e. (12), the condition for a mass-spring-damper sytem as environment is more than 10 times 'longer'. Note that all analytical calculation are done using Maple ${ }^{\mathrm{TM}}$.
}

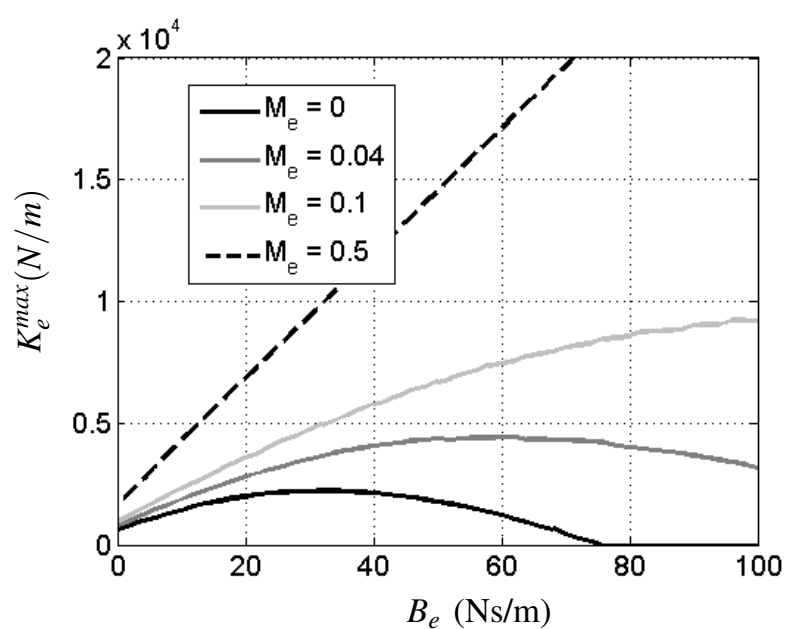

Fig. 7. The maximum value for $K_{e}$ in function of $B_{e}$ and $M_{e}$ for which $Y_{M S\left(Z_{e}\right)}$ is passive in case a low-pass-filter is used on the force-feedback channel with a $30 \mathrm{~Hz}$ bandwidth. Again, for $M_{e}=B_{e}=0$, the maximum environment stiffness is $600 \mathrm{~N} / \mathrm{m}$.

inequality in $x=\omega^{2}$. For this system, it can be calculated numerically that with $K_{e}^{\max }=600 \mathrm{~N} / \mathrm{m}, \mu \lambda$ can be maximum 0.61 . The condition $M_{e}^{\max }=0.5 \mathrm{~kg}$ does again not result in a more restrictive tuning condition. Now, in the same way as done above, it is verified whether the derived tuning condition $\mu \lambda \leq 0.61$ is a sufficient condition for all massspring-damper systems that fit into the 'infinite' box shown in Fig. 1(b). Fig. 7 shows the numerically calculated passivity condition as an upper boundary on $K_{e}$, for a range of values of $B_{e}$ and some values of $M_{e}$, for the parameters of Table I and $\mu \lambda=0.61$. Surprisingly, this figure shows that a larger environment damping does not necessarily have a relaxing effect on the upper boundary for the environment stiffness. Note that in [5] a similar finding was reported. For the case that $M_{e}=0 \mathrm{~kg}$ e.g., once the damping in the environment is larger than $35 \mathrm{Ns} / \mathrm{m}$, a further increase in damping has a negative effect on the maximum environment stiffness for which $Y_{M S}$ is passive. Moreover, for damping values $B_{e}>68 \mathrm{Ns} / \mathrm{m}$, the maximum environment stiffness becomes even smaller than the imposed boundary of $600 \mathrm{~N} / \mathrm{m}$. As such, this teleoperation system demonstrates that Assumption 1 does not generally hold.

\section{Position-force controller with a floating object}

As mentioned in the introduction, the focus in this paper is on the case where the environment can be represented by a mass-spring-damper model-structure. This was the case in the two previous examples. However, for some applications it can also be necessary to analyze the situation where the environment is e.g. a spring-damper-mass system as shown in Fig.5(b). Such a system can be seen as a representation for a floating object with a basic contact-model and the impedance of this model-structure is:

$$
Z_{e}=\frac{M_{e} B_{e} s^{2}+M_{e} K_{e} s}{M_{e} s^{2}+B_{e} s+K_{e}} .
$$

In this example it will be investigated whether the results obtained for a pure mass and pure stiffness as environment 
can be generalized to such a spring-damper-mass modelstructure. In contrast to the two previous examples, the bounded environment passivity method is used here as an analysis tool instead of a design tool: bounds on the environment are derived for a given teleoperation system, i.e. the system described in Section II. In the first example the passivity condition for a pure stiffness as environment was expressed as an upper boundary on $\mu \lambda$, the same condition is here expressed as an upper boundary for $K_{e}$ :

$$
K_{e} \leq \frac{B_{m}\left(B_{s}+K_{v}\right)}{\mu^{2} \lambda^{2} M_{s} K_{v}^{2}}\left(\mu \lambda\left(K_{v}\left(B_{s}+K_{v}\right)-2 M_{s} K_{p}\right)+2 B_{m}\left(B_{s}+K_{v}\right)+2 \sqrt{\text { Root }}\right)
$$

with Root $=$

$$
\left(B_{m}^{2}+\mu \lambda B_{m} K_{v}\right)\left(K_{v}+B_{s}\right)^{2}-\mu \lambda M_{s} K_{p} B_{m}\left(K_{v}+B_{s}\right)+\mu^{2} \lambda^{2} M_{s} K_{p}\left(M_{s} K_{p}-B_{s} K_{v}\right) .
$$

Using the parameters of Table $\mathrm{I}$, this means that $K_{e}^{\max }$ is equal to $580 \mathrm{~N} / \mathrm{m}$. As mentioned earlier, for a pure mass as environment, there is no upper boundary: $M_{e}^{\max }=\infty$. For a spring-damper-mass model-structure as environment, the positive real part condition again results in a $4^{\text {th }}$ order inequality in $x=\omega^{2}$ and thus numerical results are shown here. Fig. 8 shows the maximum environment stiffness for which $Y_{M S}$ is passive as a function of the environment mass $M_{e}$, for some values of $B_{e}$. One can see that for $B_{e}=0$ and $M_{e} \rightarrow \infty$, the maximum value for $K_{e}$ approaches the value derived for a pure stiffness. This make sense as in this situation, the spring-damper-mass resembles actually a pure stiffness fixed to the ground. However, for smaller values of $M_{e}$, the maximum value for $K_{e}$ is smaller than the value derived for a pure stiffness ${ }^{4}$. Strictly speaking, the conditions derived for a pure mass and a pure stiffness as environment can thus not be extended to a floating spring-damper-mass system. For the Position-Force controller studied here, however, Fig. 8 shows that even a very small amount of environment damping $B_{e}$, i.e. $B_{e}>0.6 \mathrm{Ns} / \mathrm{m}$ is sufficient to make the conditions derived for a pure mass and a pure stiffness valid for floating springdamper-mass systems as environment.

\section{Discussion}

The goal of this paper was to analyze two existing passivity-based methods that can take into account knowledge on the environment and/or the human operator. This knowledge should consist of possible model-structures as well as possible values or ranges of values for the different mass, spring and damper components of these modelstructures.

The first approach analyzed in this paper was the use of virtual shunt impedances when calculating absolute stability. This approach is inspired by the idea of mounting a real mechanical passive compliant element at the interaction interface of a robot that physically interacts with an environment. This idea was proposed to tackle the hard contact stability problems of force-controlled robots [14], [15], [16]. In order to affect the passivity of the interaction

\footnotetext{
${ }^{4}$ Note also that the red spot in this figure corresponds to the red spot in Fig. 4. This should be no surprise as the floating object under study here has at its interaction side a spring-damper system, which is exactly the model-structure of the shunt impedance used in Fig. 4.
}

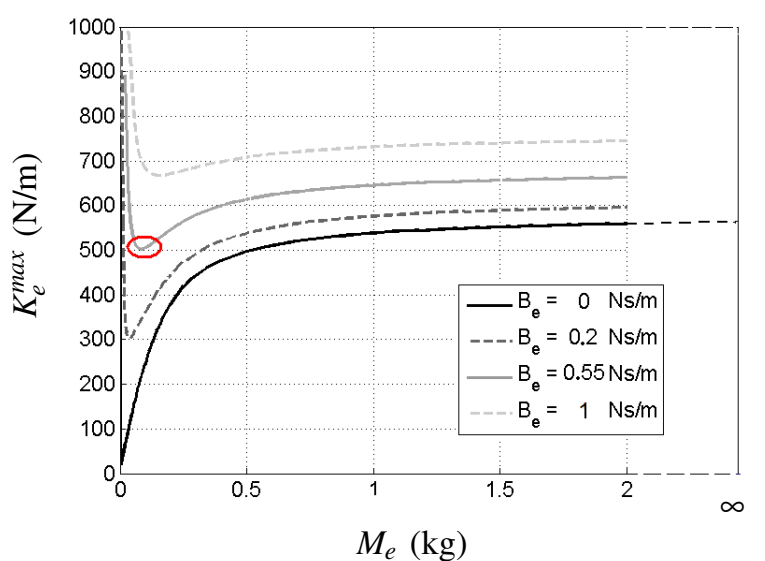

Fig. 8. Floating objects as environment: The maximum value for $K_{e}$ as a function of $M_{e}$ and $B_{e}$ for which $Y_{M S}$ is passive. For stiffnesses $>580 \mathrm{~N} / \mathrm{m}$, $Y_{M S}$ is active for any value of $M_{e}$. For stiffnesses $<580 \mathrm{~N} / \mathrm{m}$, there are values of $M_{e}$ for which the admittance $Y_{M S}$ is active.

side of such a force-controller robot, it has been shown that damping is mandatory [16], which corresponds to the finding in Section III. Based on this finding, this section showed that virtual shunt impedances cannot be used to include bounds expressed in terms of the mass, damper and stiffness when the environment/operator is assumed to be a massspring-damper system. Yet, although the usability is more restricted than originally thought, virtual shunts can be used when all possible environments and/or human operators can be characterized as having a common impedance at their interaction interface.

The second approach analyzed in this paper, bounded environment passivity, is able to include such specific knowledge on the environment when designing a teleoperation system. This knowledge can consist of possible model-structures for the environment each with its own possible value or range of values for the different mass, spring and damper components. The proposal to focus the analysis on a pure mass and a pure stiffness as environment, however, can be debated. The second example in Section IV showed that the results for a pure mass and a pure stiffness cannot necessarily be generalized to all mass-spring-damper systems with mass and stiffness values respecting the imposed/derived bounds:

- although this seems rather counter-intuitive, when the environment is assumed to be a pure damping, there can be a bound on the value for this damper: $B_{e}^{\max }$.

- for a pure mass, a pure damping and a pure stiffness cannot necessarily be generalized to all mass-springdamper systems with mass, damping and stiffness values respecting the imposed/derived bounds $M_{e}^{\max }, B_{e}^{\max }$ and $K_{e}^{\max }$. For the case that $M_{e}=0$ e.g., Fig. 6 shows that, although $K_{e}^{\max }=600 \mathrm{~N} / \mathrm{m}$ and $B_{e}^{\max }=74 \mathrm{Ns} / \mathrm{m}, Y_{M S}$ is not passive for $Z_{e}=74+\frac{600}{s} \mathrm{Ns} / \mathrm{m}$.

Therefore, to be theoretically correct, the passivity of $Y_{M S}$ should at least be verified for all eight vertices of Fig. 9(c) when the stability needs to be guaranteed for all environments within this box. Note that a similar proposition was made in [6]. In contrast to analyzing closed-loop stability of the overall system as in [6], bounded environment passivity 


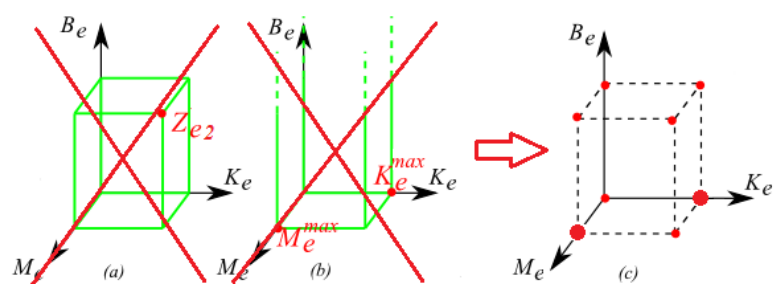

Fig. 9. To guarantee stability for all environments with a mass-springdamper model-structure and values in the $\left(M_{e}, B_{e}, K_{e}\right)$-box, one should not use the $Z_{e 2}$-shunt (a), nor focus only on bounded environment passivity for a pure mass and a pure stiffness (b) but one should look at least at the eight vertices of the box (c).

investigates the passivity of $Y_{M S}$ and as such, no assumptions on the operator are necessary, as it is generally accepted that humans can stably interact with passive systems [13].

Further research is necessary to clarify the practical implications of the above findings. For the case e.g. where $Y_{M S}$ is theoretically active for $Z_{e}=74+\frac{600}{s} \mathrm{Ns} / \mathrm{m}$, while being passive for $Z_{e}=\frac{600}{s} \mathrm{Ns} / \mathrm{m}$, will this 'being active' also result in practical noticable unstable behaviour? The authors believe that focussing on a pure stiffness or/and a pure mass during the (initial) design of a teleoperation system still makes sense. First of all, stability problems are typically noticed for hard contacts, and a pure stiffness is a good and simple model for a hard contact. Thus it allows one to focus on a problem that has clear practical implications. Furthermore, the fact that the model is kept simple facilitates analytic calcuations significantly. In [17] e.g., is shown how such analytical calculations contribute substantially to the understanding of both the transparency trade-offs and the stability-transparancy trade-off for a specific controller. This understanding for its part results in faster and smarter design optimizations.

Note that after such an (initial) design based on a pure stiffness and/or a pure mass assumption for the environment, one can still perform a robustness analysis for the more general mass-spring-damper model-structure by calculating e.g. the passivity of $Y_{M S}$ for a discretized set of points in the box of Fig. 9(c). Depending on the application, the same could be done for the spring-damper-mass modelstructure. How detailed this analysis is depends on what the designer considers as 'sufficiently' robust, taking into account the omnipresent trade-off between robustness and non-conservativeness.

\section{CONCLUSIONS}

In literature, there is a consensus that both two-port passivity and absolute stability are too conservative because both methods assume an infinite dynamic range for both the human operator and the environment. Several methods have been developed to reduce this conservatism by incorporating environment and/or operator knowledge in the controller design. In this paper we show that incorporation of such knowledge should be done with great care. This paper shows explicitly how earlier assumptions on the use of two popular methods, i.e. the use of shunt impedances [9], [10], [11], [12] and bounded environment passivity [7], [8] have to be reconsidered. The paper also shows how and for which scenarios these methods can be used in a theoretically correct way. Further research is however needed to clarify the practical implications of the obtained results.

\section{ACKNOWLEDGMENTS}

This work was supported by a $\mathrm{PhD}$ grant from the Institute for the Promotion of Innovation through Science and Technology in Flanders (I.W.T.-Vlaanderen) and by an FP7-People Marie Curie Reintegration Grant, PIRG03-2008231045. Furthermore, the authors like to thank Professor Y. Yokokohji for fruitful discussions on this topic.

\section{REFERENCES}

[1] G. R. Raju, G. Verghese, and T. Sheridan, "Design issues in 2-port network models of bilateral remote manipulation," in Proceedings of the IEEE International Conference on Robotics and Automation, vol. 3, Scottsdale, AZ, May 1988, pp. 1316-1321.

[2] D. Lawrence, "Stability and transparency in bilateral teleoperation," IEEE transactions on robotics and automation, vol. 9, no. 5, pp. 624637, October 1993.

[3] F. Llewellyn, "Some fundamental properties of transmission systems," in Proc. IRE, vol. 40, 1952, pp. 271-283.

[4] R. Adams and B. Hannaford, "Stable haptic interaction with virtual environments," IEEE transactions on robotics and automation, vol. 15 , no. 3, pp. 465-474, June 1999.

[5] A. Kron and G. Schmidt, "Stability and performance analysis of kinesthetic control architectures for bimanual telepresence systems," Journal of Intelligent and Robotic Systems, vol. 46, pp. 1-26, 2006.

[6] A. Peer and M. Buss, "Robust stability analysis of a bilateral teleoperation system using the parameter space approach," in Proceedings of IEEE International Conference on Intelligent Robots and Systems, Nice, France, September 2008, pp. 2350-2356.

[7] A. Haddadi and K. Hashtrudi-Zaad, "Bounded-impedance absolute stability of bilateral teleoperation control systems," IEEE Transactions on Haptics, vol. 3, no. 2, pp. 15-27, 2010.

[8] B. Willaert, B. Corteville, D. Reynaerts, H. V. Brussel, and E. V. Poorten, "A mechatronic analysis of the classical position-force controller based on bounded environment passivity," The Int. J. of Robotics Research, 2010.

[9] K. Hashtrudi-Zaad and S. Salcudean, "Analysis of control architectures for teleoperation systems with impedance/admittance master and slave manipulators," The international Journal of Robotics Research, vol. 20, no. 6, pp. 419-445, June 2001.

[10] R. Adams and B. Hannaford, "Control law design for haptic interfaces to virtual reality," IEEE Transactions on control systems technology, vol. 10, no. 1, pp. 3-13, January 2002.

[11] H. Cho and J. Park, "Impedance control with variable damping for bilateral teleoperation under time delay," JSME International Journal Series $C$, vol. 48, no. 4, pp. 695-703, 2005.

[12] E. Vander Poorten, "Improving haptic fidelity for intuitive manipulation in scaled teleoperation and virtual environment," $\mathrm{Ph} . \mathrm{D}$. dissertation, University of Kyoto, 2007.

[13] J. Colgate and N. Hogan, "Robust control of dynamically interacting systems," International Journal on Control, pp. 65-88, 1988.

[14] R. Roberts, R. Paul, and B. Hillberry, "The effect of wrist force sensor stiffness on the control of robot manipulators." in Proceedings of the IEEE International Conference on Robotics and Automation, 1985, pp. $269-274$

[15] D. Whitney, "Historical perspective and state of the art in robot force contol," Int. Journal of Robotics Research, vol. 6, no. 1, 1987.

[16] M. Dohring and W. Newman, "Admittance enhancement in force feedback of dynamic systems," in Proceedings of the IEEE International Conference on Robotics and Automation, Washington, DC, May 2002, pp. 638-643.

[17] B. Willaert, B. Corteville, D. Reynaerts, H. V. Brussel, and E. V. Poorten, "Transparency trade-offs for a 3-channel controller revealed by the bounded environment passivity method." in The third Int. Conf. on Advances in Computer-Human Interactions (ACHI), St. Maarten, February 2010, pp. 66-72. 